\title{
AN INNOVATION IN MOUTH CLEANSING
}

The MC3 is an ergonomically designed Class 1 medical device for use in delivering safe mouth care. Unlike brushes, it cleans the tissues and collects debris simultaneously and unlike sponge swabs, the cleaning head of the MC3 will not detach and form a safety hazard.

The MC3 was developed following a medical safety alert from the Medicines and Healthcare Products Regulatory Agency involving the use of foam mouth swabs. A foam head had detached from the stick of an oral swab while a carer was providing mouth care to an elderly patient. The foam head could not be retrieved and the patient subsequently died. Over 800 patient safety incidents have been reported by The National Patient Safety Agency where the foam head had either become detached or pieces of the foam head were torn off and retained or lodged in the mouth. Foam mouth swabs have now been banned across Wales and many Healthcare Trusts across England.

The MC3 can be used safely with ventilated patients at risk of contracting ventilated associated pneumonia (VAP). MC3 is for those people who depend on or require assistance for mouth care and for people where conventional methods such as toothbrushes and sponge swabs are not suitable.

MC3 can be used to cleanse the soft tissues inside or around the mouth for a wide range of people, including the edentulous. It can be used wet or dry to lubricate and moisten the lips and mouth with water, water based gels and mouth rinses; clean a coated tongue; remove retained food and debris from the mouth; remove sticky tenacious secretions, crusty plugs on the palate and stringy saliva; and aid oral densensitisation for patients with special requirements and learning disabilities.

The MC3 is suitable for use by any healthcare professional in hospitals, care, nursing and residential homes, hospices, community health and social service teams and in dental practices.

For more details contact Linton Whyte on 07877547762 or email lintonwhyte@live. co.uk.

\section{IMPROVE RECALL EFFECTIVENESS}

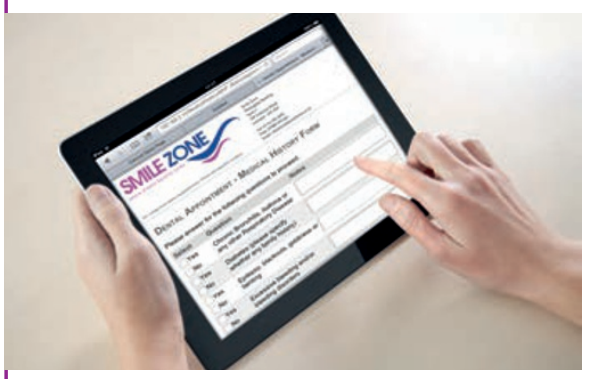

Patient recalls are often forgotten or sent only once in the hope the patient receives the message first time. Missed appointment patients are not contacted for re-booking and as a result end up in an 'idle' state with no activity in the practice. All of these issues lead to large lists of patients being registered but not being treated.

Understanding and reacting to the needs of patients is fundamental to proactive marketing. As a recognition of these issues, iSmile has a built-in Campaign Manager which can automatically generate up to three rounds of appointment reminders and up to eight rounds of recall reminders. Each message is set to be sent via any channel (SMS, email, letter or patient preference) and the message for each round of reminders can be customised. With all the activity happening automatically in the background it eases pressure on practice managers and receptionists and ensures the practice knows the state of each registered patient.

Furthermore, the iSmile system extends directly to the palm of your patients. The digital age has created a connected world with constant access to key information anywhere, anytime on any device. iSmile has an integrated Patient Portal, which can be accessed from any device and is branded to the practice's specifications. The secure Patient Portal enables the patient to complete their medical history ahead of their appointment, minimising surgery wait times and allowing the dentist to see more patients during the day.

The iSmile Patient Portal also captures patient feedback on their experience and includes a patient interest form. The feedback can then be analysed at the practice and interest specific campaigns can be setup.

For more information about iSmile call 08454681287 or visit www.ismiledental. co.uk.

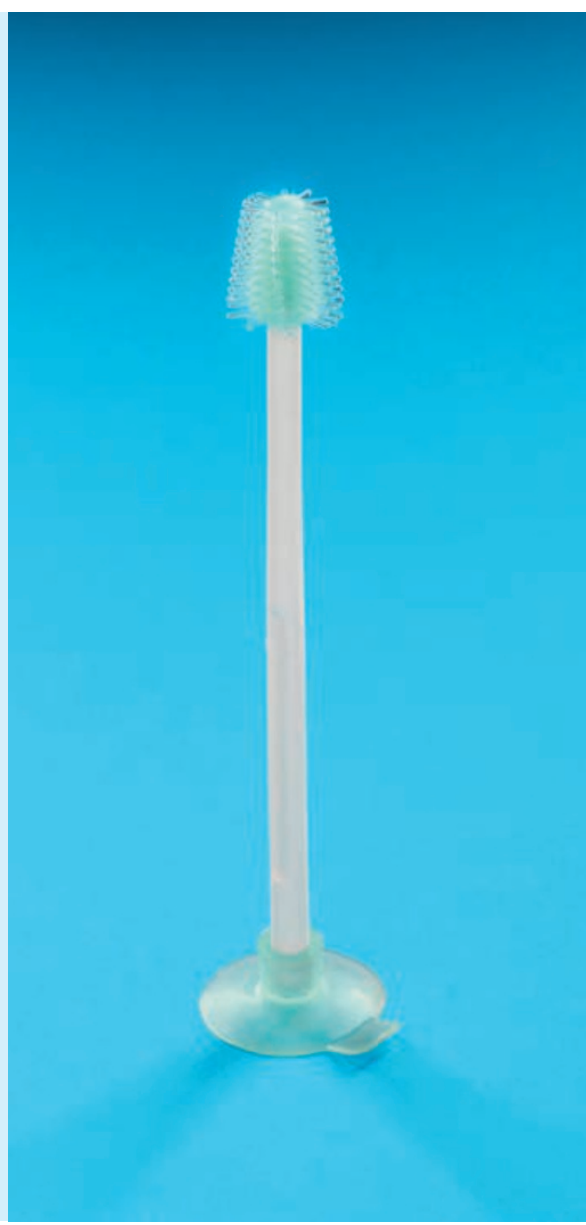

